\title{
DIVERSIDAD GENÉTICA EN CUATRO ESPECIES MEXICANAS DE CALABAZA (Cucurbita spp.)
}

\author{
GENETIC DIVERSITY IN FOUR SPECIES OF MEXICAN SQUASH (Cucurbita spp.)
}

\author{
Lourdes Cerón González ${ }^{1 *}$, Juan P. Legaria Solano², Clemente Villanueva Verduzco² y Jaime Sahagún \\ Castellanos $^{2}$
}

\begin{abstract}
${ }^{1}$ Posgrado en Horticultura y ${ }^{2}$ Departamento de Fitotecnia, Universidad Autónoma Chapingo. Km. 38.5 Carretera México-Texcoco. 56230, Chapingo, Estado de México. Tel. y Fax: 01 (595)952-1500.

* Autor para correspondencia (loce_79@yahoo.com.mx)
\end{abstract}

\section{RESUMEN}

Se analizaron colecciones de cuatro especies de calabaza cultivada (Curcubita argyrosperma Huber, C. pepo L., C. moschata (Duchesne ex Lam.) Duchesne ex Poiret, y $C$. ficifolia Bouché) provenientes de la región Centro-Sur de México, para determinar la diversidad genética entre $y$ dentro de las especies, $y$ obtener las huellas genéticas correspondientes. Se utilizaron marcadores moleculares tipo RAPD (Polimorfismos en el ADN Amplificados al Azar). Se probaron 60 iniciadores y se estudió un total de 185 loci. El porcentaje de loci polimórficos entre especies fue 90.6\%. Dentro de cada especie hubo reducida variabilidad genética, con porcentajes de $14.7 \%$ en $C$. argyrosperma, $14 \%$ en $C$. ficifolia, $20.8 \%$ en $C$. pepo y $37 \%$ en $C$. moschata. El grado de flujo genético fue bajo $(\mathrm{Nm}=$ 0.14) lo que indica que hay menos de un migrante por generación entre las poblaciones de las especies. El coeficiente de diferenciación genética entre las poblaciones $(\mathrm{Gst}=\mathbf{0 . 7 7})$ indicó que las cuatro especies están altamente diferenciadas. Los marcadores RAPD agruparon las especies en cuatro grandes grupos, correspondientes a cada una de las especies. $C$. argyrosperma y $C$. moschata fueron las especies más relacionadas con un coeficiente de identidad de 0.79. $C$. pepo se relacionó con $C$. argyrosperma y $C$. moschata con coeficiente de identidad de 0.63 y 0.69 , respectivamente, y la especie más alejada fue $\boldsymbol{C}$. ficifolia.

Palabras clave: Curcubita argyrosperma, C. ficifolia, C. pepo, C. moschata, RAPD, diversidad genética.

\section{SUMMARY}

Accessions of four Mexican species of squash (Curcubita argyrosperma Huber, C. pepo L., C. moschata (Duchesne ex Lam.) Duchesne ex Poiret, and $\boldsymbol{C}$. ficifolia Bouché) from Central-Southern México were analyzed to determine genetic diversity among and within species, and to obtain the corresponding genetic fingerprints. RAPD (Random Amplified Polymorphic DNA) molecular markers were used. Sixty primers were tested, and 185 loci were studied. Percentage of polymorphic loci among species was $90.6 \%$. Low genetic variability was detected within species, accounting for 14.7 $\%$ of polymorphism in C. argyrosperma, $14 \%$ in C. ficifolia, $20.8 \%$ in $C$. pepo and $37 \%$ in $C$. moschata. Genetic flow was present at low levels $(\mathrm{Nm}=\mathbf{0 . 1 4})$, indicating the occurrence of less than one migrant per generation among populations of species. The coefficient of genetic differentiation among population $(\mathrm{Gst}=\mathbf{0 . 7 7})$ showed that the four species are highly differentiated. RAPD markers grouped the species into four large groups, each species corresponding to one group. $C$. argyrosperma and $C$. moschata were the most related species with an identity coefficient of 0.79 . C. pepo was related to $C$. argyrosperma and $C$. moschata with identity coefficients of 0.63 and 0.69 , respectively. $C$. ficifolia was the most distant species.

Index words: Curcubita argyrosperma, C. ficifolia, C. pepo, C. moschata, RAPD, genetic diversity.

\section{INTRODUCCIÓN}

La familia Cucurbitaceae incluye alrededor de 90 géneros y 750 especies. El género Cucurbita, uno de los más importantes, cuenta con 27 especies (Whitaker, 1974; Hernández, 1978). Las especies de este género forman el grupo conocido como calabazas, de las cuales cinco han sido domesticadas: $C$. pepo L. (calabaza de india), $C$. ficifolia Bouché (chilacayote), C. moschata (Duchesne ex Lam.) Duchesne ex Poiret (calabaza de castilla); $C$. maxima Duchesne ex Lam (calabaza kabosha) y $C$. argyrosperma Huber. Con excepción de C. maxima las calabazas se encuentran ampliamente distribuidas en México, en pequeña escala $C$. maxima se encuentra en Centroamérica y parte norte de América del Sur (Hernández, 1978; Lira-Saade, 1995).

El número básico de cromosomas de todas las especies de Cucurbita es $2 \mathrm{n}=2 \mathrm{x}=40$ y los cariotipos sugieren que estas especies son de origen alopoliploide (Singh, 1979; Weeden, 1984; Lebeda et al., 2007).

La adaptación ambiental de las especies es la siguiente: C. moschata en lugares cálidos, con altitud menor de $1000 \mathrm{~m}$; C. pepo en lugares con altitud superior a 1000 $\mathrm{m}$; C. ficifolia en altitudes mayores de $1300 \mathrm{~m}$ (Whitaker, 1968); y C. máxima en lugares con clima templado. 
C. argyrosperma es una especie cultivada pero también puede encontrarse en estado silvestre. Su área de distribución como especie abarca desde el suroeste de los Estados Unidos hasta Nicaragua, generalmente en zonas por debajo de los $1800 \mathrm{~m}$, con climas cálidos y algo secos (Villanueva, 2007).

En México la variación genética es muy amplia, principalmente en forma, tamaño y coloración del fruto, cantidad de semilla producida, calidad y cantidad de pulpa, tolerancia a enfermedades y precocidad (Kohashi, 1960; Whitaker, 1968; Montes, 1991). En estos caracteres, en México se encuentran variabilidad genética importante en $C$. pepo y $C$. moschata, y en un nivel inferior en C. ficifolia y C. mixta (Hernández, 1978).

A pesar de su alto grado de diferenciación, ninguna de las especies del género Cucurbita está completamente aislada en términos reproductivos. Experimentos de hibridación revelan que entre las especies cultivadas $C$. moschata tiene el más alto grado de compatibilidad con C. argyrosperma. El siguiente nivel de compatibilidad se presenta entre $C$. argyrosperma y $C$. pepo y con algunos cultivares de C. máxima (Lira-Saade, 1995).

Estudios isoenzimáticos revelan moderada diferenciación genética dentro de C. pepo y C. moschata, mientras que poca diferenciación ha ocurrido dentro de $C$. argyrosperma, C. ficifolia y C. máxima (Andres, 1990; Decker, 1988; Decker-Walters et al., 1990).

La diversidad de $C$. argyrosperma es menor que la de C. pepo, C. moschata, y C. máxima (Lira-Saade, 1995). C. moschata es una especie altamente polimórfica, con considerable diversidad morfológica en semillas y frutos (Andres, 2004). C. maxima y $C$. pepo muestran variabilidad molecular y morfológica semejante (Ferriol et al., 2004; Decker-Walters et al., 2002), mientras que la variación genética y morfológica es muy limitada en C.ficifolia (Andres, 1990; Lebeda et al., 2007).

La caracterización de las colectas se ha basado frecuentemente en el empleo de caracteres morfológicos o agronómicos. El uso de marcadores morfológicos presenta algunas limitantes en la caracterización, pues su expresión puede estar sujeta a factores ambientales o fenológicos. Con frecuencia estos marcadores pueden ser evaluados a nivel de toda la planta y cuando ésta llega a su estado adulto. Para los investigadores el tomar los datos de las plantas hasta alcanzar el estado adulto representa una espera que se refleja en retraso. Actualmente se han desarrollado métodos de identificación y caracterización basados en el uso de marcadores moleculares que superan, en la mayoría de casos, las limitantes de los métodos tradicionales (Azofeita Delgado, 2006). En México se carece de estudios a nivel molecular que estimen la diversidad genética del género Cucurbita y de sus especies cultivadas.

El objetivo del presente trabajo fue estimar la diversidad genética presente entre y dentro de especies de Cucurbita cultivadas en México, así como determinar si el análisis de los patrones RAPD puede ser útil para la identificación de colecciones y especies.

\section{MATERIALES Y MÉTODOS}

Se evaluaron poblaciones base en la forma de compuestos balanceados (100 semillas por familia incluida) para cada una de las cuatro especies cultivadas de calabaza. Las correspondientes poblaciones de calabaza se integraron con 7, 20, 3 y 20 familias (colectas originales provenientes de 1996 que fueron previamente sometidas a cuatro ciclos de incremento y adaptación a las condiciones de Chapingo Edo. de México) de C. ficifolia, C. moschata, $C$. pepo y $C$. argyrosperma, respectivamente. Las colectas originales provenían de los Estados de Morelos, Nayarit, Oaxaca, Veracruz, Yucatán y Estado de México.

Los marcadores RAPD se obtuvieron en 40 plantas por especie (a los 25 d después de la germinación) crecidas en invernadero. El ADN genómico se purificó con el método propuesto por De la Cruz et al. (1997), que consiste en: macerar en nitrógeno líquido, $0.3 \mathrm{~g}$ de tejido de hojas jóvenes y frescas, sin dejar descongelar, posteriormente el pulverizado se mezcló mediante agitación por $60 \mathrm{~s}$ dentro de un microtubo (Eppendorf) de $1.5 \mathrm{~mL}$ con $600 \mu \mathrm{L}$ de amortiguador de extracción (20 mL de Tris- $\mathrm{HCl} 1 \mathrm{M}, \mathrm{pH}$ 8.0; $20 \mathrm{~mL}$ de EDHTA $0.5 \mathrm{M}, \mathrm{pH} 8.0 ; 20 \mathrm{~mL} \mathrm{NaCl} 5$ $\mathrm{M} ; 35 \mu \mathrm{l}$ de $\beta$-mercaptoetanol; $40 \mathrm{~mL}$ de dodecil sulfato de sodio $20 \%$ ), luego se incubó a $65{ }^{\circ} \mathrm{C}$ por $10 \mathrm{~min}$, con inversión ocasional de los tubos. Después se adicionan $200 \mu \mathrm{L}$ de acetato de potasio $5 \mathrm{M}$, se agitó por inversión y se incubó 30 min en hielo, luego se centrifugó a 18000 $\mathrm{x} g$ durante $10 \mathrm{~min}$ a temperatura ambiente, $\mathrm{y}$ el sobrenadante se transfirió a otro microtubo que contenía $700 \mu \mathrm{L}$ de isopropanol frío $\left(-20{ }^{\circ} \mathrm{C}\right)$. Se mezcló por inversión, se incubó a $-20{ }^{\circ} \mathrm{C}$ durante $30 \mathrm{~min}$ y se centrifugó por $5 \mathrm{~min}$ a $8000 \mathrm{x} \mathrm{g}$, a temperatura ambiente. El sobrenadante se eliminó, se recuperó el precipitado y se disolvió en $200 \mu \mathrm{L}$ de solución (Tris- $\mathrm{HCl} 50 \mathrm{mM}$, EDTA-Na2 $10 \mathrm{mM}$, pH 8.0).

Para eliminar el ARN se utilizaron $2 \mu \mathrm{L}$ de ARNasa A, a $37{ }^{\circ} \mathrm{C}$ durante $1 \mathrm{~h}$. Después se adicionaron $20 \mu \mathrm{L}$ de acetato de sodio a una concentración de 3 M y $200 \mu \mathrm{L}$ de 
isopropanol, se mezcló por inversión y se dejó precipitar a $-20{ }^{\circ} \mathrm{C}$ por $2 \mathrm{~h}$. Se centrifugó por 5 min a $8000 \mathrm{x}$ g, a temperatura ambiente. Se eliminó el sobrenadante y se lavó el precipitado con $300 \mu \mathrm{L}$ de etanol $70 \%$, se secó la pastilla y se disolvió en $100 \mu \mathrm{L}$ de amortiguador TE (Tris - $\mathrm{HCl} 10 \mathrm{mM}$, EDTA-Na2 $1 \mathrm{mM}, \mathrm{pH}$ 8.0) a una temperatura de $4{ }^{\circ} \mathrm{C}$.

La concentración del ADN se cuantificó en un espectrofotómetro Jenway 6305 UV/Vis ${ }^{\circledR}$ (Barloworld Scientific, England.) y la calidad se verificó por electroforesis en geles de agarosa a $1.2 \%(\mathrm{p} / \mathrm{v})$. El ADN se usó para llevar acabo las reacciones en cadena de la Taq ADN polimerasa (PCR, por sus siglas en inglés). Se probaron 20 iniciadores de la serie A de Operon ${ }^{\circledR}$ (OPA01-OPA20), 20 de la serie B (OPB01-OPB20) y 20 de la serie D (OPD01-OPD20) (Operon Technologies Inc, Alameda, CA, USA.). De ellos se seleccionaron 19 que mostraron polimorfismo, y por la complejidad del patrón de bandeo de los RAPD (Cuadro 1). La PCR se realizó en un termociclador Perkin Elmer Modelo $480 \AA$ (News Jersey, USA.). La mezcla de reacción se hizo en un volumen total de $25 \mu \mathrm{L}$, que incluyó: $4.2 \mu \mathrm{L}$ de agua bidestilada estéril, $10 \mu \mathrm{L}$ de dNTPs $(500 \mu \mathrm{M}), 2.5 \mu \mathrm{L}$ de amortiguador 10X Tris- $\mathrm{HCl} 750 \mathrm{mM}, \mathrm{pH} 8.8 ;\left(\mathrm{NH}_{4}\right)_{2} \mathrm{SO}_{4}$ $200 \mathrm{mM}$; Tween 20 a $1 \%(\mathrm{v} / \mathrm{v}) ; 1.0 \mu \mathrm{L}$ de $\mathrm{MgCl}_{2}(50$ $\mathrm{mM}) ; 3.0 \mu \mathrm{L}$ del iniciador a una concentración de $10 \mathrm{pM}$; $0.3 \mu \mathrm{L}$ de enzima Taq ADN polimerasa a una

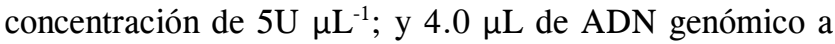
una concentración de $10 \mathrm{ng} \mu \mathrm{L}^{-1}$.

Las condiciones de reacción fueron: un ciclo a $94{ }^{\circ} \mathrm{C}, 2$ min; 38 ciclos $\left[94{ }^{\circ} \mathrm{C}\right.$ por $30 \mathrm{~s}, 40{ }^{\circ} \mathrm{C}$, por $30 \mathrm{~s}, 72{ }^{\circ} \mathrm{C}$ por $90 \mathrm{~s}$; ; al final $72{ }^{\circ} \mathrm{C}$ por $2 \mathrm{~min}$. La separación de los fragmentos amplificados se hizo por electroforesis en geles de agarosa a $1.2 \%(\mathrm{p} / \mathrm{v})$ con amortiguador TAE (40 $\mathrm{mM}$ Tris-acetato, $\mathrm{pH} 7.6 ; 1 \mathrm{mM} \mathrm{Na} 2$ EDTA), durante $3 \mathrm{~h}$ a $85 \mathrm{~V}$. Los geles se tiñeron con bromuro de etidio $(0.5$ $\mathrm{mg} \mathrm{mL} \mathrm{m}^{-1}$ ) durante $15 \mathrm{~min}$, después se eliminó el exceso de colorante con agua destilada estéril por 15 min más y se fotografiaron bajo luz UV. Las reacciones de amplificación se repitieron al menos dos veces, sin que se presentaran discrepancias entre los patrones de bandeo obtenidos.

Los materiales se compararon con base en similitudes y diferencias en los patrones de bandeo. Se asignó valor de 1 para la presencia de banda y 0 para la ausencia. Se construyó una matriz de datos en una hoja de cálculo para obtener un dendrograma de relaciones entre las colectas con el coeficiente de Jaccard (Jaccard, 1908) y con el método de agrupamiento UPGMA (Unweighted Pair Group Method using Arithmetic Averages). Se hizo un análisis de remuestreo ("Bootstrapping", 1000 repeticio- nes) con el fin de obtener datos numéricos de consistencia del árbol generado con el programa estadístico Free Tree versión 0.9.1.50 (Hampl et al., 2001).

El porcentaje de loci polimórficos se calculó mediante la fórmula Nei (1973): Porcentaje de loci polimórficos $=$ número de loci polimórficos/número total de loci $x 100$. Se consideró a un locus como polimórfico cuando la frecuencia del alelo más frecuente fue inferior o igual a $95 \%$.

Cada especie fue considerada como una población para estimar parámetros de estructura poblacional, al purificar ADN de 40 muestras individuales de cada especie. La matriz de datos presencia/ausencia de bandas se analizó con el programa POPGENE32 (Yeh et al., 1999). Los parámetros de polimorfismo estimados dentro y entre poblaciones fueron: porcentaje de loci polimórficos (P), número de alelos por locus $(\mathrm{A})$, número efectivo de alelos por locus (Ae), índice de Shannon (S), índice de diversidad genética de Nei $(\mathrm{H})$, coeficiente de diferenciación genética (Gsт) y número de individuos migrantes $(\mathrm{Nm})$. El coeficiente de diferenciación genética entre poblaciones (Gst) se calculó como: $G_{S T}=D_{S T} / H_{T}$, donde $H_{T}$ es la diversidad genética total y $D_{S T}$ es la proporción de la diversidad genética de las especies que está presente entre las poblaciones (Nei, 1973); este coeficiente permite determinar la proporción de diversidad genética que se distribuye entre poblaciones. El número de individuos migrantes $(\mathrm{Nm})$ se calculó como: $N m=1 / 4\left(1 / G_{s T}-1\right)$, donde $G_{S T}$ es el coeficiente de diferenciación genética entre poblaciones. $\mathrm{Nm}$ da una idea del grado de flujo genético entre las poblaciones (Nei, 1973). El número efectivo de alelos por locus $(A e)$ es el número de alelos que determinan la heterocigosidad u homocigosidad observada, y se calcula como: $A e=1 / \Sigma p i^{2}$, donde $p i$ es la frecuencia del i-ésimo alelo.

\section{RESULTADOS Y DISCUSIÓN}

De 60 iniciadores probados de las series A, B y D de Operon ${ }^{\circledR}, 19$ presentaron productos de amplificación (Cuadro 1). La media de polimorfismo detectada por los iniciadores fue de $89.05 \%$. Los iniciadores OPA06, OPA09, OPA15, OPA19, OPD02, OPD12 у OPD18 revelaron $100 \%$ de polimorfismo. El número promedio de bandas reveladas por iniciador fue de 9.73, con oscilación entre 4 y 16, y se estudió un total de 185 loci. 
Cuadro 1. Lista de iniciadores y sus secuencias, número de productos amplificados, polimorfismo detectado por especie y total.

\begin{tabular}{|c|c|c|c|c|c|c|c|}
\hline \multirow{2}{*}{ Iniciador } & \multirow{2}{*}{ Secuencia } & \multirow{2}{*}{ Prod. amplificados } & \multicolumn{4}{|c|}{ Polimorfismo por especie (\%) } & \multirow{2}{*}{$\begin{array}{c}\text { Polimorfismo } \\
\text { total }(\%)\end{array}$} \\
\hline & & & C. argyrosperma & C. ficifolia & C. moschata & C. pepo & \\
\hline OPA02 & 5'-TGCCGAGCTG-3' & 10.0 & 0.0 & 10.0 & 0.0 & 0.0 & 80.0 \\
\hline OPA03 & 5'-AGTCAGCCAC-3' & 16.0 & 18.0 & 0.0 & 6.2 & 6.2 & 87.0 \\
\hline OPA04 & 5'-AATCGGGCTG-3' & 8.0 & 0.0 & 12.5 & 37.0 & 37.0 & 75.0 \\
\hline OPA05 & 5'-AGGGGTCTTG-3' & 10.0 & 40.0 & 40.0 & 50.0 & 20.0 & 90.0 \\
\hline OPA06 & 5'-GGTCCCTGAC-3' & 4.0 & 50.0 & 25.0 & 50.0 & 25.0 & 100.0 \\
\hline OPA09 & 5'-GGGTAACGCC-3' & 9.0 & 33.3 & 0.0 & 22.0 & 44.0 & 100.0 \\
\hline OPA11 & 5'-CAATCGCCGT-3' & 10.0 & 0.0 & 20.0 & 10.0 & 10.0 & 90.0 \\
\hline OPA13 & 5'-CAGCACCCAC-3' & 11.0 & 0.0 & 0.0 & 9.0 & 9.0 & 90.0 \\
\hline OPA15 & 5'-TTCCGAACCC-3' & 7.0 & 0.0 & 0.0 & 42.0 & 0.0 & 100.0 \\
\hline OPA19 & 5'-CAGGCCCTTC-3' & 4.0 & 0.0 & 25.0 & 0.0 & 0.0 & 100.0 \\
\hline OPA18 & 5'-AGGTGACCGT-3' & 11.0 & 0.0 & 0.0 & 45.0 & 27.0 & 90.0 \\
\hline OPD02 & 5'-GGACCCAACC-3' & 7.0 & 14.0 & 0.0 & 42.0 & 0.0 & 100.0 \\
\hline OPD03 & 5-GTCGCCGTC A-3 & 13.0 & 7.6 & 23.0 & 30.0 & 15.0 & 69.0 \\
\hline OPD08 & 5'-GTGTGCCCCA-3' & 13.0 & 7.6 & 38.0 & 30.0 & 15.0 & 92.0 \\
\hline OPD11 & 5'-AGCGCCATTG-3' & 10.0 & 0.0 & 10.0 & 10.0 & 10.0 & 80.0 \\
\hline OPD12 & 5'-CACCGTATCC-3' & 5.0 & 40.0 & 0.0 & 0.0 & 20.0 & 100.0 \\
\hline OPD13 & 5'-GGGGTGACGA-3' & 10.0 & 10.0 & 30.0 & 10.0 & 30.0 & 80.0 \\
\hline OPD18 & 5'-GAGAGCCAAC-3' & 14.0 & 0.0 & 0.0 & 14.0 & 28.0 & 100.0 \\
\hline OPD20 & 5'-ACCCGGTCAC-3' & 13.0 & 7.6 & 7.6 & 15.0 & 7.6 & 69.0 \\
\hline Promedio & & 9.73 & 12.0 & 12.7 & 22.2 & 15.98 & 89.0 \\
\hline
\end{tabular}

El análisis basado en la técnica RAPD permitió estimar la similitud y diferencia genómica entre los genotipos en estudio. En la Figura 1 se muestran los patrones de bandas obtenidos con los iniciadores OPA03, OPA13, OPD03 y OPD08, donde se evidencia la existencia de variabilidad genética entre y dentro de las especies evaluadas.

En la Figura 2 se muestra un dendrograma de relaciones de similitud entre colecciones pertenecientes a diferentes especies de Cucurbita obtenido mediante el análisis de 1000 remuestreos con el programa Free Tree Ver. 0.9.1.50. Las colecciones de cada especie se agruparon juntas sin que ocurriera mezcla entre las mismas, lo que significa que los marcadores RAPD fueron adecuados para diferenciarlas. Los resultados muestran la formación de cuatro grupos, que corresponden a las cuatro especies evaluadas. $C$. moschata y $C$. argyrosperma fueron las especies genéticamente más emparentadas, con un coeficiente de similitud de 0.53. $C$. pepo divergió a un coeficiente de similitud de Jaccard de 0.33 , mientras que $C$. ficifolia fue la especie genéticamente más alejada ( 0.26 de coeficiente de similitud). En lo referente a colecciones dentro de cada especie, las más similares correspondieron a C. ficifolia, C. argyrosperma y $C$. pepo, y las menos similares fueron las de $C$. moschata (Figura 2). Estos resultados concuerdan con los de Lira-Saade (1995) y Andrés (1990, 2004) para caracteres morfológicos, en lo referente a las relaciones de similitud que guardan entre sí las especies estudiadas y al grado relativo de variación dentro de cada especie.

El porcentaje de loci polimórficos (P) entre las especies fue de $90.60 \%$ (Cuadro 2). C. argyrosperma mostró un valor de polimorfismo de $14.77 \%$, C. ficifolia $14.09 \%$, C. moschata $37.58 \%$ y C. pepo $20.81 \%$. Estos valores indican también que las poblaciones presentan baja variabilidad genética. Los resultados coinciden con los de Andres (2004) quien indica que $C$. moschata es una especie altamente polimórfica, y con los de Lira-Saade (1995) en el sentido de que $C$. argyrosperma presenta menor diversidad genética que C. pepo, C. moschata y $C$. máxima. Según Lira-Saade (1995), C. argyrosperma es menos diversa que $C$. pepo, C. moschata y C. máxima. Andres (2004) encontró que C. moschata muestra considerable diversidad morfológica en semillas y frutos. $C$. maxima y $C$. pepo muestran niveles semejantes de diversidad molecular y morfológica (Ferriol et al., 2004; Decker-Walters et al., 2002) mientras que la variación genética y morfológica es muy limitada en $C$. ficifolia (Andres, 1990).

El coeficiente de diversidad $(\mathrm{H})$ de Nei (1973) para todos los loci estudiados en las cuatro especies de Cucurbita fue de 0.4727 (Cuadro 2); por especie, los coeficientes fueron: 0.063 para $C$. argyrosperma, 0.058 
para $C$. ficifolia, 0.116 para $C$. moschata y 0.104 para $C$. pepo. Tanto estos valores como los porcentajes de loci polimorficos, reflejan poca diversidad genética en las poblaciones.

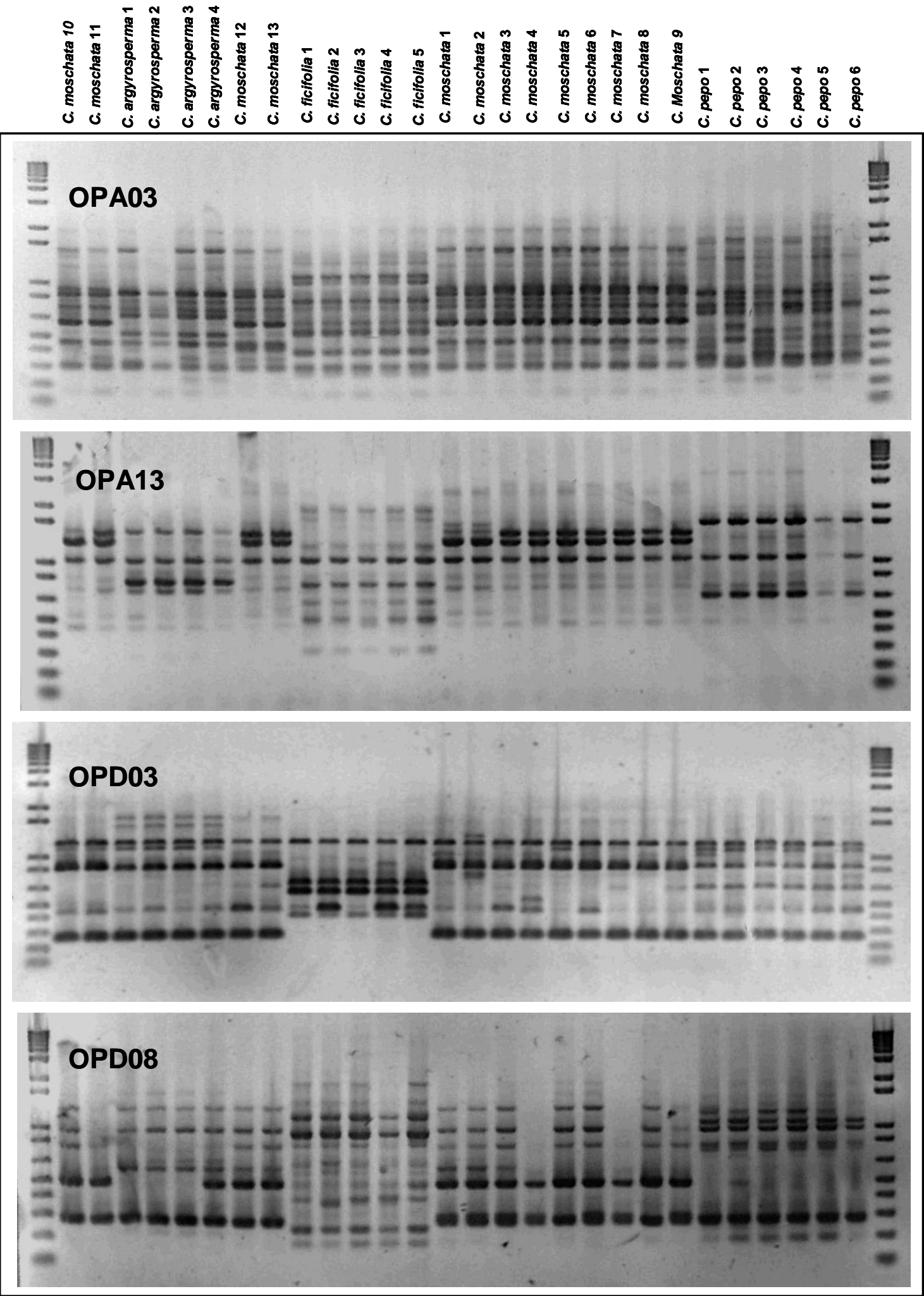

Figura 1. Patrones de bandas RAPD obtenidos con los iniciadores OPA03, OPA13, OPD03 y OPD08 en colecciones de cuatro especies de Cucurbita separados por electroforesis en gel de agarosa a $1.2 \%$. 


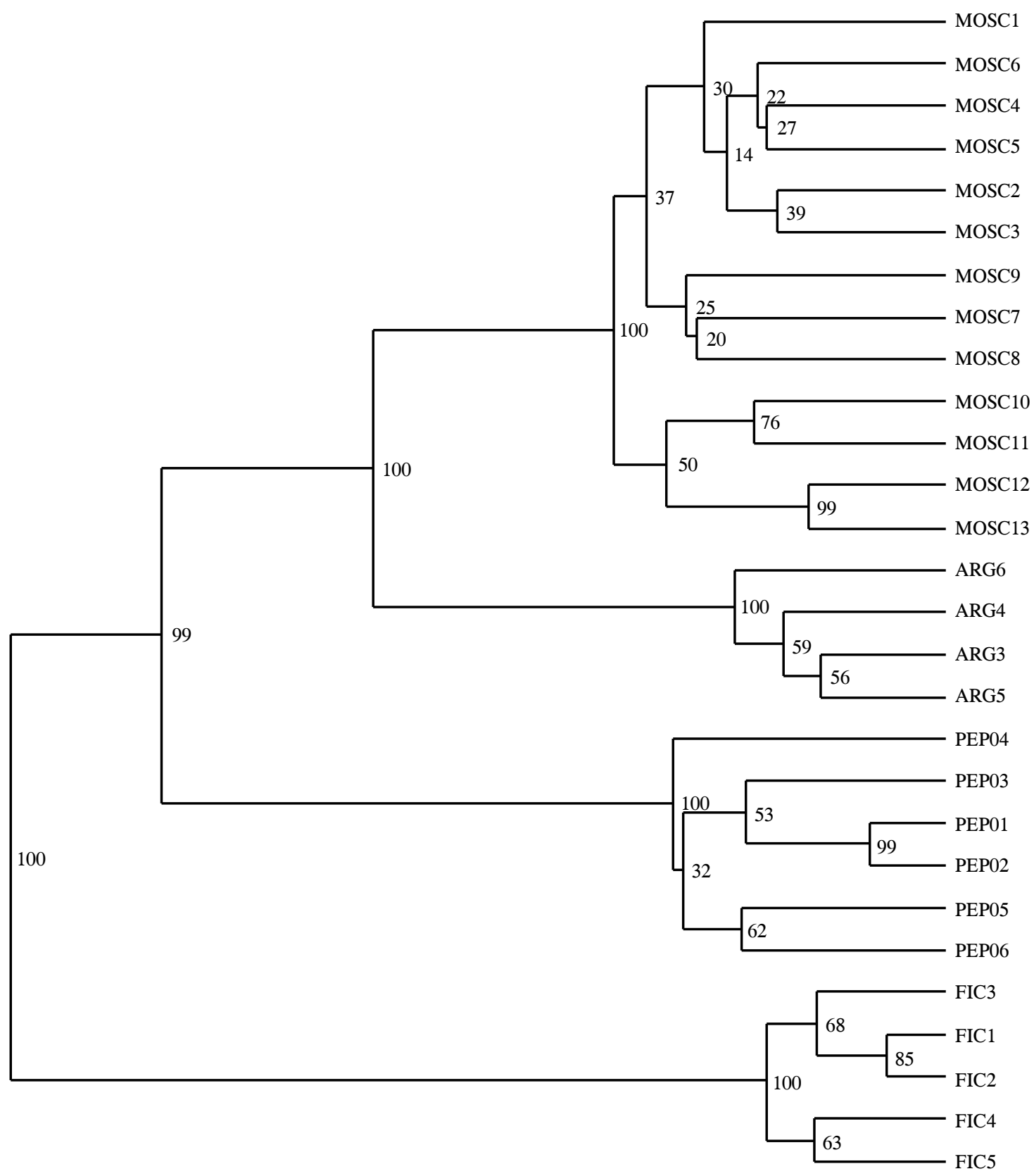

0.1

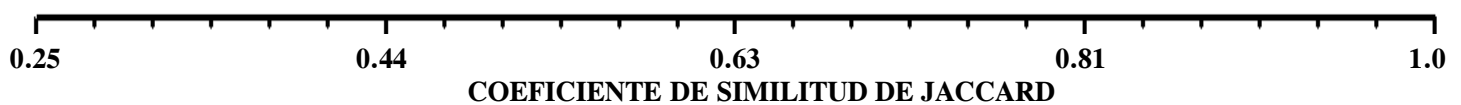

Figura 2. Dendrograma construido a partir de 1000 remuestreos obtenidos de los patrones de bandeo de RAPD de colecciones de $C$. argyrosperma, C. pepo, $C$. moschata, y $C$. ficifolia, mediante el coeficiente de Jaccard y el método de agrupamiento UPGMA. La línea inferior indica 0.1 de disimilitud entre las especies. Los valores en las ramas indican el porcentaje de ocurrencia de la topología de una rama particular durante el análisis de robustez del árbol. 
En lo referente al número de alelos por locus (A) y al número efectivo de alelos por locus (Ae), C. moschata superó a las especies restantes con valores de 1.37 y 1.20 , respectivamente. El bajo valor de diversidad genética de $C$. argyrosperma con respecto a $C$. moschata y $C$. pepo puede deberse a que las variedades locales de la primera especie han evolucionado en un área geográfica bastante restringida (Lira-Saade, 1995). C. moschata, que mostró los valores más altos de diversidad genética se cultiva en un amplio rango de alturas y debido a ello ha adquirido adaptación a varias condiciones ambientales. La baja variabilidad genética de C. ficifolia según Andres (2004) pudiera estar asociada con la existencia de escasas variedades criollas; su escasa variabilidad morfológica es consistente con su limitado grado de variabilidad isoenzimática.

El coeficiente de diferenciación genética entre las especies fue alto $(G s T=0.77)$, lo que indica que aproximadamente $77 \%$ de la variación detectada se debe a diferencias entre especies. El resto $(23 \%)$ representa diversidad genética dentro de especies. Andrés (1990), Decker (1988) y Decker-Walters et al. (1990) también encontraron niveles de diferenciación genética de moderada a escasa dentro de cada especie de Cucurbita. Es posible que las especies domesticadas presenten algún grado de aislamiento reproductivo provocado por factores geográficos, genéticos, fisiológicos, morfológicos o culturales.

Con base en el coeficiente de diferenciación genética total entre las especies (GST) el grado de flujo genético $(\mathrm{Nm})$ estimado fue 0.14 (Cuadro 2). Esto significa que hay menos de un individuo migrante por generación entre las poblaciones, lo que podría explicar también su alto grado de diferenciación.

En la Figura 3 se muestra un dendrograma de relaciones de similitud entre especies de Cucurbita resultante del análisis de poblaciones. $C$. moschata, $C$. argyrosperma y $C$. pepo son más similares entre sí. $C$. moschata fue más similar con $C$. argyrosperma a un coeficiente de identidad de 0.79 , con $C$. pepo a 0.69 , y con C. ficifolia a 0.53. C. argyrosperma se relacionó con C. pepo y $C$. ficifolia a 0.63 y 0.50 de identidad, respectivamente, mientras que $C$. pepo y $C$ ficifolia relacionaron con un valor de 0.56 .

Cuadro 2. Diversidad genética dentro y entre especies de Cucurbita spp.

\begin{tabular}{|c|c|c|c|c|c|c|c|}
\hline Población & $\mathrm{P}(\%)$ & A & $\mathrm{Ae}$ & $S$ & $\mathrm{H}$ & Gst & $\mathrm{Nm}$ \\
\hline C. argyrosperma & 14.77 & $1.148(0.36)$ & $1.116(0.29)$ & $0.091(0.22)$ & $0.063(0.16)$ & & \\
\hline C. ficifolia & 14.09 & $1.141(0.35)$ & $1.106(0.28)$ & $0.085(0.21)$ & $0.058(0.15)$ & & \\
\hline C. pepo & 20.81 & $1.208(0.41)$ & $1.113(0.26)$ & $0.068(0.15)$ & $0.104(0.21)$ & & \\
\hline Entre especies & 90.60 & $1.906(0.29)$ & $1.527(0.32)$ & $0.314(0.15)$ & $0.473(0.21)$ & 0.774 & 0.146 \\
\hline
\end{tabular}

$\mathrm{P}=$ porcentaje de loci polimórficos; $\mathrm{A}=$ número de alelos por locus; $\mathrm{Ae}=$ número efectivo de alelos por locus; $\mathrm{S}=$ coeficiente de diversidad de Shannon; $\mathrm{H}=$ coeficiente de diversidad de Nei; Gst = coeficiente de diferenciación genética entre poblaciones; Nm = grado de flujo génico. Los valores entre paréntesis indican la desviación estándar.
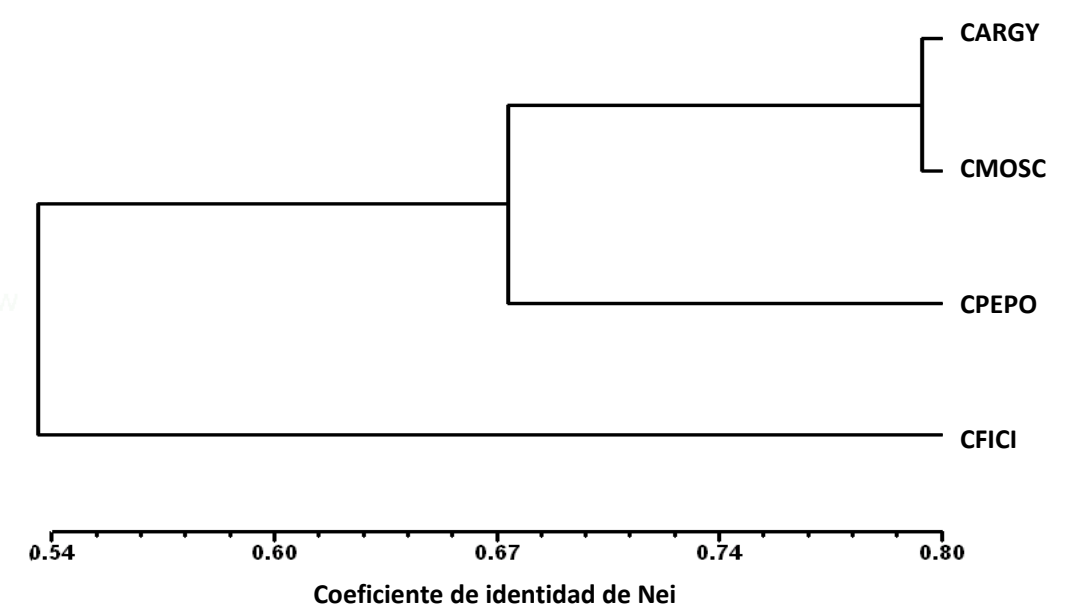

Figura 3. Dendrograma de similitud entre especies de Cucurbita, obtenidas con la fórmula de Nei (1973). 
Lo expuesto sugiere que los marcadores RAPD son eficientes para discriminar colecciones y especies de Cucurbita, lo que concuerda con la clasificación morfológica y molecular con marcadores AFLP (amplified fragment length polymorphisms) y SRAP (sequence-related amplified polymorphisms) establecida por otros autores (Lira-Saade, 1995; Ferriol et al., 2004).

\section{CONCLUSIONES}

Existió variabilidad genética dentro y entre especies de Cucurbita evaluadas en este trabajo, la mayor parte de la diversidad genética detectable se encuentra entre especies $y$ en menor proporción dentro de especies. Cucurbita moschata se relacionó más fuertemente con $C$. argyrosperma y la especie menos similar fue C. ficifolia. Las especies mostraron poca diversidad genética interna. La especie con mayor polimorfismo genético fue $C$. moschata y la que mostró más bajo fue $C$. ficifolia.

\section{BIBLIOGRAFÍA}

Andres T C (1990) Biosystematics, theories on the origin and breeding potential of Cucurbita ficifolia. In: Biology and Utilization of the Cucurbitaceae. D M Bates (ed). Comstock Publishing Associates. Ithaca, N.Y. pp:102-119.

Andres T C (2004) Diversity in tropical pumpkin (Cucurbita moschata): a review of intraspecific classifications. In: Progress in Cucurbit Genetics and Breeding Research. Proc. of Cucurbitaceae 2004, 8th EUCARPIA Meeting on Cucurbit Genetics and Breeding. A Lebeda, H S Paris (eds). Olomouc, Czech Republic. pp:107-112.

Azofeita-Delgado AA (2006) Uso de marcadores moleculares en plantas; aplicaciones en frutales del trópico. Agron. Mesoam. 17:221-242.

Decker D S (1988) Origin(s), evolution, and systematics of Cucurbita pepo (Cucurbitaceae). Econ. Bot. 42:4-15.

Decker-Walters D S, T W Walters, U Poluszny, P G Kevan (1990) Genealogy and gene flow among annual domesticated species of Cucurbita. Can. J. Bot. 68:782-789.
Decker-Walters D S, J E Staub, S M Chung, E Nakata, H D Quemada (2002) Diversity in free-living populations of Cucurbita pepo (Cucurbitaceae) in North America. Plant Syst. Evol. 233:183-197.

De la Cruz M, F Ramirez, H Hernandez (1997) DNA isolation and amplification from cacti. Plant Mol. Biol. Rep. 15:319-325.

Ferriol M, B Picó, P Fernández de Córdova, F Nuez (2004) Molecular diversity of a germplasm collection of squash (Cucurbita moschata) determined by SRAP and AFLP markers. Crop Sci. 44:653-664.

Hampl V, A Pavlícek, J Flegr (2001) Construction and bootstrap analysis of DNA fingerprinting-based phylogenetic trees with the freeware program Free Tree: application to trichomonad parasites. Intl. J. Syst. Evol. Microbiol. 5:731-735.

Hernández B G (1978) Cucurbitaceas. In: T Cervantes S (ed). Recursos Genéticos Disponibles a México. SOMEFI. Chapingo, México. $\mathrm{pp}: 357-367$.

Jaccard P (1908) Nouvelles recherches sur la distribution florale. Bull. Soc. Vaudoise Sci. Nat. 44:223-70.

Kohashi S J (1960) Mejoramiento genético de la calabaza (Cucurbita spp.) en México. Proc. A.S.H.S. R. Trop. 4:16-19.

Lebeda A, M P Widrlechner, J Staub, H Ezura, J Zalapa, E Křistová (2007) Cucurbits (Cucurbitaceae; Cucumis spp., Cucurbita spp., Citrullus spp.) In: Genetic Resources, Chromosome Engineering, and Crop Improvement. Vol. 3. Vegetable Crops. R J Singh (ed). CRC Press. Boca Raton, FL, USA. pp:271-376.

Lira-Saade R (1995) Estudios Taxonómicos y Ecogeográficos de las Cucurbitáceas Latinoamericanas de Importancia Económica. IPGRI. Roma, Italia. $281 \mathrm{p}$.

Montes H S (1991) Calabazas (Cucurbita spp.). In: Avances en el Estudio de los Recursos Fitogenéticos de México. R Ortega $\mathrm{P}$, G Palomino H, F Castillo G, V A González H, M Livera M (eds). SOMEFI. Chapingo, México. pp:239-250.

Nei M (1973) Analysis of gene diversity in subdivided populations. Proc. Nat. Acad. Sci. USA. 70:3321-3323.

Singh A K (1979) Cucurbitaceae and polyploidy. Cytologia 74:897-905.

Villanueva V C (2007) Calabazas Cultivadas. Identificación de Especies, Caracterización y Descripción Varietal. Universidad Autonoma Chapingo. Chapingo, Edo. de México. 123 p.

Weeden N F (1984) Isozyme studies indicate that the genus Cucurbita is an ancient tetraploid. Cucurbit Genet. Coop. Rep. 7:84-85.

Whitaker T W (1968) Ecological aspects of the cultivated Cucurbita. HortScience 3:9-11.

Whitaker T W (1974) Cucurbita. In: Handbook of Genetics. R C King (ed). Plenum Press. New York, USA. pp:135-144.

Yeh F C, R-C Yai, T Boyle (1999) Popgene Version 1.31. Microsoft Window-based Freeware for Population Genetic Analysis. Quick User Guide. Edmonton, Alberta, Canada. 28 p. 